\title{
Forced Convective Heat Transfer over Ribs at Various Separation
}

\author{
Chun-Ho Liu ${ }^{1}$, Tracy N.H. Chung \\ Department of Mechanical Engineering, The University of Hong Kong, \\ Pokfulam Road, Hong Kong, China.
}

\begin{abstract}
Flow over transverse ribs is a fundamental problem that has numerous applications in a range of scales from turbine cooling to urban roughness. It can be broadly divided into $k$-type or $d$-type flows exhibiting different characteristics. In this study, large-eddy simulation (LES) is used to examine the flows between two ribs at various separation and compare the local heat transfer coefficient (LHTC) on the cavity bottom. Flow instability initiates a dividing streamline at the leeward edge. In $k$-type flows, it reattaches at the cavity bottom that splits into a leeward recirculation and a windward redeveloping turbulent boundary layer (TBL). Heat removal from the recirculation and the redeveloping TBL is governed by intermittency and entrainment, respectively. Moreover, the dividing streamline impinges on the cavity bottom leading to a local maximum of LHTC. In $d$-type flows, the dividing streamline covers the cavity isolating the recirculation from the prevailing flow aloft. Heat is therefore solely removed by intermittency in which the LHTC increases monotonically from the leeward to the windward sides.
\end{abstract}

\footnotetext{
${ }^{1}$ Tel: (852) 2859 7901; Fax: (852) 2858 5415; E-mail: liuchunho@graduate.hku.hk
} 


\section{Introduction}

Cavities flanked by ribs occur in many flow configurations, by design or circumstances, for a range of scales such as finned cooling of electronic devices, turbine flow passages, or urban roughness. Boundary-layer separation and reattachment over cavities of different rib-height-to-cavity-width (aspect) ratios result in various flow features modifying the drag and heat transfer which has been a popular research problem for decades [1].

In fluid mechanics, flows over a rough wall with two-dimensional (2D) transverse ribs are broadly grouped into $k$ - or $d$-type depending on the aspect ratio [2]. Similarly, flows over idealized 2D street canyons are divided into isolated roughness, wake interference, and skimming flow regimes for urban climatic studies [3]. In view of the analogous transport equations of heat and mass, we study the heat transfer over transverse ribs in this paper and attempt to elucidate the transport processes over street canyons.

Early studies, assuming uniform heat flux or constant temperature at the cavity bottom, have focused on the local heat transfer coefficient (LHTC) of an isolated cavity. Simulating a constant temperature solid boundary, water evaporation was used to determine the correlation between LHTC and aspect ratio [4]. Including a channel of finite depth over a cavity, the lowest LHTC is measured at the windward corner that increases monotonically toward the leeward side for both $k$ - and $d$-type flows [5]. Moreover, the channel depth does not affect much the LHTC at the cavity bottom, laying down the basic domain requirement for the studies of heat and mass transfer in cavities.

Apart from an isolated cavity, laboratory experiments have been employed elucidating the LHTC on repeated ribs in which 2 to 3 more ribs are 
added upwind facilitating fully developed flows in and over the sample cavity [6]. The heat transfer over repeated ribs of aspect ratio 1/10 was improved by a factor of 2 to 3 than that over a smooth surface [7]. Some other ribs of different shapes and aspect ratios were also tested elsewhere [8, 9].

In the light of heat and mass transfer analogy, experiments have been conducted by the urban climate community to examine the fluxes of atmospheric constituents from urban areas diffusing upward into the atmospheric boundary layer (ABL). The LHTCs over $2 \mathrm{D}$ ribs of aspect ratios in the range of $1 / 2$ to 2 were measured by naphthalene sublimation [10] and water evaporation [11]. Whereas, a major discrepancy is observed between the experimental results that is likely caused by the large difference in Reynolds number and Schmidt number [12].

Similar to most turbulence studies, computational fluid dynamics (CFD) have been commonly adopted in heat transfer because of their fully controllable parameters and detailed data output in a transient manner. The Reynolds-averaged Navier-Stokes (RANS) approach offers reasonably accurate solutions in a timely manner [13] that has been widely employed in the sensitivity tests of flows and heat transfer to rib geometry and aspect ratio [14]. Ribs of a wider range of aspect ratio were tested recently using RANS $k-\epsilon$ turbulence model to depict the characteristic flows and temperature distributions in different flow regimes [15]. In particular, the maxima of heat transfer coefficient and drag force overlap with each other as a function of aspect ratio $[16,17]$.

More insights for the detailed turbulent transport processes over 2D ribs have been revealed using detached-eddy simulation (DES), direct numeri- 
cal simulation (DNS), and large-eddy simulation (LES). While DES is able to capture both mean and secondary flows, RANS turbulence model often fails in LHTC calculation because of the less accurate computations of separation zone and flow reattachment [18]. DNS has been used by several research groups to derive the correlation among aspect ratio, flows, and heat transfer over periodic ribs. For ribs of aspect ratio $1 / 8$ in $k$-type flows, the characteristic near-wall streaky flow patterns are clearly identified by DNS [19]. Moreover, the higher-order turbulence statistics are very localized in the vicinity to the ribs [20]. On the other hand, in $d$-type flows, the near-wall streaky flow structures are less elongated than those over a smooth wall [21]. Infinitely repeated ribs of other aspect ratios were tested differentiating the characteristic flows and heat transfer in $k$ - and $d$-type flows, from which the more pronounced heat transfer performance was found in $k$-type flows over $2 \mathrm{D}$ ribs $[22]$.

LES has been actively sought in the urban climate community to determine the turbulent flows and fluxes over urban areas, which, however, are more focused on $d$-type flows because of the small building separation. The vertical fluxes over $2 \mathrm{D}$ ribs of various aspect ratios were compared to formulate the pollutant removal mechanism [23] and examine the turbulence scales [24]. Recently, the flow instabilities at the leeward rib is identified in [25], arousing our interests in differentiating the roles of mean flows and turbulence in the heat and mass transfer from 2D infinitely repeated ribs.

A series of LES studies have been performed by the authors to examine the characteristic flows and LHTC over periodic 2D ribs of different aspect ratios in the urban climate perspective [26]. It was found that the flow separates 
at the leeward rib and impinges on the cavity bottom at the reattachment point in $k$-type flows. The reattached flow is then split into an upstream flow and a downstream flow along cavity bottom. The flow upstream is a turbulent recirculation between the leeward rib and the reattachment. The flow downstream after the reattachment point redevelops a turbulent boundary layer (TBL) then separates again passing over the windward rib. On the other hand, in $d$-type flows, the separation between the ribs is small so the separated flow does not reattach down to the cavity bottom but connects the ribs. A recirculation is thus developed spinning the entire cavity instead.

The LHTCs in $k$ - and $d$-type flows were thoroughly analyzed [26], whereas, the flow mechanism leading to such behaviors is yet fully understood. This paper is thus conceived to enrich our knowledge of the flows, and heat and mass transfer over 2D ribs using LES. This section outlines the background of the problem. The mathematical model and the methodology are detailed in Section 2. Section 3 demonstrates how to manage the LHTC at different Reynolds number and Section 4 validates the current LES with other experimental data sets available in literature. The LES results, both mean and transient properties, are diagnosed in Section 5.

\section{Mathematical Model}

LES is used in this study to examine the LHTC on the cavity bottom. The open-source CFD code OpenFOAM [27] is employed and the methodology is described in this section. 


\subsection{Governing Equations}

An incompressible flow in isothermal condition is assumed that consists of the continuity

$$
\frac{\partial \bar{u}_{i}}{\partial x_{i}}=0
$$

and the momentum conservation

$$
\frac{\partial \bar{u}_{i}}{\partial t}+\frac{\partial}{\partial x_{j}} \bar{u}_{i} \bar{u}_{j}=-\Delta \mathrm{P} \delta_{i 1}-\frac{\partial \bar{p}}{\partial x_{i}}+\nu \frac{\partial^{2} \bar{u}_{i}}{\partial x_{j} \partial x_{j}}-\frac{\partial \tau_{i j}}{\partial x_{j}} .
$$

Tensor notation with summation convention on repeated indices $(i, j)$ is adopted. The prevailing flow is driven by the background kinematic pressure gradient $\Delta \mathrm{P}$ which is switched on only in the part of domain over the ribs. The Reynolds stress in Equation (2), which accounts for the subgrid-scale (SGS) transport, is modeled using the Smagorinsky model [28]

$$
-\tau_{i j}=-\nu_{\mathrm{SGS}}\left(\frac{\partial \bar{u}_{i}}{\partial x_{j}}+\frac{\partial \bar{u}_{j}}{\partial x_{i}}\right)+\frac{2}{3} k_{\mathrm{SGS}} \delta_{i j}
$$

together with the conservation of SGS turbulence kinetic energy (TKE) $k_{\mathrm{SGS}}$

$$
\frac{\partial k_{\mathrm{SGS}}}{\partial t}+\frac{\partial}{\partial x_{i}} k_{\mathrm{SGS}} \bar{u}_{i}=-\frac{1}{2} \tau_{i j} \frac{\partial \bar{u}_{i}}{\partial x_{j}}+\left(\nu+\nu_{\mathrm{SGS}}\right) \frac{\partial^{2} k_{\mathrm{SGS}}}{\partial x_{i} \partial x_{i}}-\mathrm{C}_{\epsilon} \frac{k_{\mathrm{SGS}}^{3 / 2}}{\Delta} .
$$

Given a small temperature difference in a high-speed flow, the buoyancy is negligible and the resolved-scale temperature $\bar{\theta}$ is calculated as a passive scalar using the advection-diffusion equation

$$
\frac{\partial \bar{\theta}}{\partial t}+\frac{\partial}{\partial x_{i}} \bar{\theta} \bar{u}_{i}=\alpha \frac{\partial^{2} \bar{\theta}}{\partial x_{i} \partial x_{i}}-\frac{\partial \gamma_{i}}{\partial x_{i}},
$$

in which the SGS heat flux $\gamma_{i}$ is modeled using eddy diffusivity

$$
\gamma_{i}=-\alpha_{\mathrm{SGS}} \frac{\partial \bar{\theta}}{\partial x_{i}} \text {. }
$$

112 In this paper, the LES is presented as a heat transfer problem. In fact, the temperature and the Prandtl number can be switched to scalar mixing ratio and Schmidt number $(\mathrm{Sc})$, respectively, for mass transfer problems. 


\subsection{Computational Domain and Boundary Conditions}

Similar to [29], the current LES computational domain is a single unit of cavity flanked by two identical ribs of height $h$ placed apart at separation $b$ (Figure 1). We are aware of the possibility of insufficient number of ribs for fully developed flows in the open channel. A prior test was performed and realized that the flows inside the cavity are not affected too much regardless using single, 3,12 , or 36 cavities of unity aspect ratio. Hence, only one cavity is modeled in the LES as we focus on the properties on the cavity bottom. The top of the leeward and windward ribs extends $0.5 h$, respectively, in the upwind and downwind directions. The flow boundary condition (BC) is periodic in the streamwise direction constructing infinitely repeating ribs along the flow. Aloft the ribs is an open channel of height $5 h$. The channel lengths are $5 h, 12 h$, and $16 h$ for the ribs of aspect ratios $1 / 4,1 / 11$, and $1 / 15$, respectively. The domain top is thermally insulated at which Neumann conditions are applied for the velocity and pressure. The LES domain is homogeneous in the spanwise direction representing a pair of infinitely long ribs. Its spanwise extent is $5 h$ in both the cavity and the open channel where periodic BCs are applied. No-slip boundaries are applied on the rib surfaces and the cavity bottom, which are heated up uniformly to a constant temperature $\Theta_{h}$. The inflow is prescribed at a constant background temperature $\Theta_{c}$, assuming $\Theta_{h}$ is higher than $\Theta_{c}$ in the analysis. Hot air is carried away from the domain by the prevailing flow using a non-reflective open $\mathrm{BC}$ of temperature $\partial \bar{\theta} / \partial t+\bar{u} \partial \bar{\theta} / \partial x=0$ at the outflow. 


\subsection{Wall Parametrization}

Solving directly the laminar sublayer is unaffordable, the law of the wall

$$
z^{+}=u^{+}+\frac{1}{\mathrm{E}}\left\{e^{\kappa u^{+}}-\left[1+\kappa u^{+}+\frac{1}{2}\left(\kappa u^{+}\right)^{2}+\frac{1}{6}\left(\kappa u^{+}\right)^{3}\right]\right\}
$$

[30] is thus adopted to model the no-slip boundaries in the current LES. It is solved iteratively for $u_{\tau}$ to calculate $\nu_{\mathrm{SGS}}$ in the near-wall region

$$
\nu_{\mathrm{SGS}}=\frac{u_{\tau}^{2}}{\left|\nabla \bar{u}_{||} \cdot \hat{n}\right|}-\nu .
$$

Equations (7) and (8) essentially implement

$$
u^{+}= \begin{cases}z^{+} & \text {If } z^{+} \text {is small (laminar sublayer) } \\ \frac{1}{\kappa} \ln \left(\mathrm{E} z^{+}\right) & \text {If } z^{+} \text {is large (logarithmic region) }\end{cases}
$$

which is consistent with the standard wall treatment [31].

\subsection{Numerical Method}

The LES domains are discretized into over 6.3 million elements by the finite volume method (Table 1) and are integrated in time using the secondorder-accurate backward difference. The meshes are stretched in the wallnormal direction so higher spatial resolution is applied near the solid boundaries to resolve the large velocity gradient. The Gaussian integration with linear interpolation scheme (second-order-accurate central difference) is used in the spatial derivatives. The implicit coupling between pressure and velocity in incompressible flows is solved by the pressure implicit with splitting operator (PISO). The (symmetric) linear equation system of pressure is solved by the preconditioned conjugate gradient (PCG) method while the rest by the preconditioned bi-conjugate gradient (PBiCG) method. 
Table 1: Spatial resolution of the LES computational domain.

\begin{tabular}{cll} 
Aspect ratio $h / b$ & Spatial resolution $\mathrm{N}_{x} \times \mathrm{N}_{y} \times \mathrm{N}_{z}=$ total elements \\
\hline $1 / 15$ and $1 / 11$ & open channel & $480 \times 150 \times 50=6,300,000$ \\
& cavity & $450 \times 150 \times 40$ \\
\hline $1 / 4$ & open channel & $650 \times 150 \times 50=7,575,000$ \\
& cavity & $450 \times 150 \times 40$ \\
\hline
\end{tabular}

In this paper, the prevailing flow speed $U$ is the velocity scale, the rib height $h$ the length scale, and the temperature difference $\Delta \Theta\left(=\Theta_{h}-\Theta_{c}\right)$ the temperature scale. Hence, the time scale is $h / \mathrm{U}$. The Reynolds number in the $k$ - and $d$-type flows equals 127,000 and 101,715 , respectively.

\section{Data Reduction}

LHTC $\omega\left(=q_{\text {conv }} /(\mathrm{U} \Delta \Theta)\right)$ is partitioned into four components

$$
q_{\mathrm{conv}}=\langle\bar{w}\rangle\langle\bar{\theta}\rangle+\left\langle w^{\prime \prime} \theta^{\prime \prime}\right\rangle+\left\langle\alpha_{\mathrm{SGS}} \frac{\partial \bar{\theta}}{\partial z}\right\rangle+\alpha \frac{\partial\langle\bar{\theta}\rangle}{\partial z}
$$

in the current LES. On the right-hand side of Equation (10), the first term is the heat flux carried by mean flow, the second the resolved-scale turbulent heat flux, the third the SGS heat flux, and the last the molecular heat flux. The conductive heat flux is $q_{\text {cond }}=\rho \mathrm{C}_{\mathrm{p}} \alpha \Delta \Theta / h$, hence,

$$
\mathrm{Nu}=\frac{\omega \mathrm{U} \Delta \Theta}{\rho \mathrm{C}_{\mathrm{p}} \alpha(\Delta \Theta / h)}=\frac{\omega h \mathrm{U}}{\rho \mathrm{C}_{\mathrm{p}} \alpha} .
$$

that is used to compare the augmented heat transfer.

A few data sets are used to validate the current LES. Because of the different Reynolds number, the LHTC is further normalized to eliminate 
scale effects. Heat transfer data are often correlated empirically as [32]

$$
\mathrm{Nu}=c \operatorname{Re}^{m} \operatorname{Pr}^{n} .
$$

For flows over a flat plate, $m$ and $n$ are around $4 / 5$ and $1 / 3$, respectively [33]. Assuming $m$ and $n$ remain unchanged for the flows over ribs that yields

$$
c=\frac{\mathrm{Nu}}{\operatorname{Re}^{m} \operatorname{Pr}^{n}}=\omega \frac{h \mathrm{U}}{\rho \mathrm{C}_{\mathrm{p}} \alpha \mathrm{Re}^{m} \operatorname{Pr}^{n}},
$$

which is supposed to be a function of the rib geometry. This dimensionless LHTC is used to examine the forced convective heat transfer behaviors.

\section{Model Validation}

The $d$-type [2] or skimming flow [3] regime is characterized by a large primary recirculation being isolated from the prevailing flow. The current LEScalculated LHTC is monotonically increasing from the leeward to windward sides that agrees well with the experimental result collected in a laboratoryscale duct [34] (Figure 2a). Probably due to the coarser measurement resolution, the corner troughs of LHTC, which are attributed to the flow impingement on the ribs, are not differentiated in the experiment.

The LHTC calculated by $k-\epsilon$ model is obviously overpredicted compared with that of the experiment [34] (Figure 2a). This discrepancy is likely caused by the implicit weaknesses of $k-\epsilon$ models handling recirculating flows and the anisotropic turbulence at the cavity top under strong shear. Hence, RANS modeling results should be interpreted with cautions.

Increasing the rib separation to $h / b=1 / 11$ changes to the $k$-type [2] or isolated roughness [3] regime that exhibits a different spatial pattern of 
LHTC (Figure 2b). The flow separates at the leeward rib and reattaches at the cavity bottom at $x / h=5[35]$, developing a primary recirculation behind the leeward rib. After the reattachment point, the prevailing flow entrains into the cavity for the TBL re-development on the windward side, separates again near the windward rib at $x / h=11$ and leaves the cavity eventually.

The experimental result [34] and the current LES are comparable with each other in $k$-type flows as well. Similar to the ribs of $h / b=1 / 4$, the minimum LHTC right at the cavity corners, which is induced by flow impingement, is only resolved by the LES. Besides, a broad maximum of LHTC is found in $3 h<x<5 h$ that is a result of the reattachment impinging the cavity bottom. The mechanism underneath is detailed in the next section.

Different from its $h / b=1 / 4$ counterpart, the LHTC along the bottom of the cavity of $h / b=1 / 11$ calculated by $k$ - $\epsilon$ turbulence model is more comparable with the experimental and LES results. Their improved agreement in $k$-type flows is likely attributed to the wider rib separation so the recirculation on the leeward side covers less than $1 / 4$ of the cavity. As such, the uncertainty of RANS models does not affect too much the LHTC of the entire cavity. It is noteworthy that heat and mass removal from pedestrian level in dense urban areas often falls in skimming flow regime, ( $d$-type flow), hence, RANS results should be applied cautiously in flows over urban roughness.

\section{Results and Discussion}

In this section, the flows for the ribs of $h / b=1 / 15$ and $1 / 4$ are analyzed, respectively, for the heat transfer mechanism in $k$-and $d$-type flows over idealized $2 \mathrm{D}$ roughness. The reattachment point is more distinguished in a 
wider cavity, hence, $h / b=1 / 15$ is reported in this section instead of $1 / 11$.

\subsection{Mean Flow and Local Heat Transfer Coefficient}

\subsection{1. k-type flows}

In $k$-type flows, the rib separation is wide enough such that the prevailing flow aloft can entrain down into the cavity bottom (Figure 3). Figure 3a shows the LHTC at the bottom of the cavity of $h / b=1 / 15$. Passing over the leeward edge, a persistent clockwise-rotating primary recirculation is developed behind the leeward rib (Figure $3 b$ ) that is consistent with some other experimental [35] and modeling [36] results over ribs in channels or pipes [37]. The streamline after the leeward flow separation reattaches the cavity bottom at $x=5 h$ that completely encloses the primary recirculation forming a reversely flowing wall jet [38]. The stagnant recirculation core thus weakens the heat removal near the rib on the leeward side.

Behind the reattachment point, the flow is partly driven by the prevailing wind entrainment after which a TBL is developed in the cavity. The mean flow in the re-developing TBL promotes the forced convective heat removal as well. Owing to the blockage of windward rib, the flow separates at $x=$ $15 h(=0.5 h$ measuring from the windward rib) then leaves the cavity. The impingement on the windward rib leads to the peaked LHTC at the corner.

\subsection{2. d-type flows}

Reducing the rib separation results in the $d$-type flows over idealized roughness elements (Figure 4). The rib clearance is narrow thus the prevailing wind no longer entrains into the cavity, and the flow reattachment and separation vanish. A large clockwise-rotating primary recirculation occupies 
the entire cavity so hot air accumulates on the leeward side. The LHTC is also closely coupled to the mean flow structures (Figure 4a). It increases monotonically from the leeward to the windward side because of the vigorous flow intermittency near the windward rib (Figure 4b).

\subsection{Turbulence Structures}

The turbulence structures also contribute substantially to the LHTC. Generally, the prevailing wind over the ribs is pressure driven while the flow inside the cavity is shear driven. The large velocity difference between the flows is the major mechanism governing the turbulent heat removal.

\subsection{1. k-type flows}

Figure 5 shows the root-mean-square (RMS) velocities and turbulent kinetic energy (TKE) in the cavity of $h / b=1 / 15$. The strong velocity shear between the prevailing wind and the leeward clockwise-rotating recirculation cascades TKE locally. Broad maxima of RMS velocities are therefore observed right over the leeward recirculation. It is noteworthy that the TKE is peaked around $x=4 h$ close to the maximum LHTC.

The concept of dividing streamline [39] commonly used in urban climatic studies is adopted in this study to examine how the recirculating flows affect turbulent heat transfer. The dividing streamline is clearly illustrated in Figure 3b impinging the cavity bottom. Its rapid divergence leads to strong dispersion and the local maximum of LHTC. Afterward, it is diverted into either the reversely flowing wall jet on the leeward side or the re-developing TBL on the windward side. Air masses originated below the dividing streamline enter into the leeward recirculation. Simultaneously, air masses over the 
dividing streamline entrain into the windward re-developing TBL. The dividing streamline induces strong velocity shear that overlaps with the peak RMS velocities, carrying the locally produced turbulence into the cavity.

Additional turbulent transport properties are revealed by the current LES. Turbulence production in isothermal condition is the product of momentum flux $\left\langle u_{i}^{\prime \prime} u_{j}^{\prime \prime}\right\rangle$ and the mean velocity gradient $\partial\left\langle\bar{u}_{i}\right\rangle / \partial x_{j}$. For example, the production term of the vertical RMS velocity $\left\langle w^{\prime \prime} w^{\prime \prime}\right\rangle^{1 / 2}$ is

$$
P=-\left\langle u^{\prime \prime} w^{\prime \prime}\right\rangle \frac{\partial\langle\bar{u}\rangle}{\partial z} .
$$

Similar to most TBL flows, the vertical momentum flux $\left\langle u^{\prime \prime} w^{\prime \prime}\right\rangle$ is negative (Figure 6a) because of its downward transport from the prevailing flow to the cavity bottom. Only a tiny amount of upward vertical momentum flux is found at the leeward corner that is a result of the reverse flows in the recirculation. The minimum vertical momentum flux is located along the cavity top at $x=4 h$, suggesting that the momentum mainly entrains right over the dividing streamline following the descending prevailing flow. Combining with the strong shear initiated at the leeward rib, a layer of strong turbulence production is thus observed on the leeward side overlapping with the dividing streamline.

The cavity bottom is hot thus the temperature variance is high (Figure $6 \mathrm{~b})$. Hot air is carried to the leeward rib following the clockwise-rotating recirculation so the temperature variance is peaked in the leeward wake. Likewise, hot air leaves the cavity following the prevailing wind entrainment then moves into the re-developing TBL. Because of the flow impingement, the temperature variance is peaked again on the windward rib.

The streamwise heat flux $\left\langle\theta^{\prime \prime} u^{\prime \prime}\right\rangle$ is mostly negative, representing heat 
dispersion in the streamwise direction by mean flow (Figure 6c). A narrow positive $\left\langle\theta^{\prime \prime} u^{\prime \prime}\right\rangle$ is observed in the wake behind the leeward rib that is a result of reverse flows in the recirculation. Hence, the streamwise heat flux tends to re-distribute the heat accumulated near the leeward rib to the core of recirculation in which the temperature is more uniform. Another crest of $\left\langle\theta^{\prime \prime} u^{\prime \prime}\right\rangle$ is found at the windward rib due to the abrupt flow impingement and separation.

Heat is transferred upward in the entire cavity as depicted by the positive vertical heat flux $\left\langle\theta^{\prime \prime} w^{\prime \prime}\right\rangle$ (Figure 6d). The maximum $\left\langle\theta^{\prime \prime} w^{\prime \prime}\right\rangle$ over the leeward rib is attributed to the flow separation and the associated intermittency. It is rather uniform in the rest of the cavity because of the wide rib separation. The vertical heat flux near the leeward rib is small, demonstrating the dominance of heat transfer by mean flows.

\subsection{2. d-type flows}

Different from its $k$-type counterpart, the maximum turbulence intensities of $d$-type flows in the cavity of $h / b=1 / 4$ are shifted from the leeward to the windward sides (Figure 7). The mean streamwise velocity gradient $\partial\langle\bar{u}\rangle / \partial z$ is peaked on the leeward side, implying that the elevated steamwise RMS velocity $\left\langle u^{\prime \prime} u^{\prime \prime}\right\rangle^{1 / 2}$ is not only attributed to the local velocity gradient.

The current LES is homogeneous in the spanwise direction so the mean spanwise velocity gradient $\partial\langle\bar{v}\rangle / \partial z$ equals zero. As such, the spanwise RMS velocity is not directly produced by velocity shear but is received from pressure-strain interactions. Different from its streamwise counterpart, $\left\langle v^{\prime \prime} v^{\prime \prime}\right\rangle^{1 / 2}$ is peaked along the windward rib coinciding with the downward wall jet (Figure $7 \mathrm{~b}$ ). This marked increase in $\left\langle v^{\prime \prime} v^{\prime \prime}\right\rangle^{1 / 2}$ is thus largely associated 
with the form drag on the windward rib.

In view of the fully enclosed dividing streamline in $d$-type flows, vertical RMS velocity $\left\langle w^{\prime \prime} w^{\prime \prime}\right\rangle^{1 / 2}$ plays a key role removing heat from the cavity through the shear layer by intermittency. It is peaked at the windward cavity top (Figure 7c), facilitating turbulent entrainment into the cavity along the windward rib. Similar to its spanwise counterpart, the form drag on the windward rib promotes TKE cascade so $\left\langle w^{\prime \prime} w^{\prime \prime}\right\rangle^{1 / 2}$ is elevated locally. TKE is peaked on the windward side that demonstrates the substantial contribution from turbulence to the vigorous intermittent entrainment along the windward rib in $d$-type flows (Figure $7 \mathrm{~d}$ ).

Similar to $k$-type flows, the vertical momentum flux $\left\langle u^{\prime \prime} w^{\prime \prime}\right\rangle$ in $d$-type flows is mostly negative in the cavity, suggesting the dominance of downward turbulent momentum transport from the prevailing flow to the cavity (Figure 8a). The maximum $\left\langle u^{\prime \prime} w^{\prime \prime}\right\rangle$ resides on the windward cavity top overlapping with the maximum TKE, hence, turbulence in the cavity is partly attributed to the descending momentum in the large scales in the prevailing flow. The vertical momentum flux then entrains down into the cavity bottom following the wall jet. It is positive and more uniform on the leeward side, thus, turbulence is largely carried by the mean flow recirculation from the windward to the leeward sides.

The temperature variance $\left\langle\theta^{\prime \prime} \theta^{\prime \prime}\right\rangle$ is peaked near the leeward rib (Figure $8 \mathrm{~b})$ so the temperature $\langle\bar{\theta}\rangle$ is more uniform because of the more complete mixing. Moreover, the temperature distribution in $d$-type flows is more uniform than its $k$-type counterpart.

The minimum streamwise heat flux $\left\langle\theta^{\prime \prime} u^{\prime \prime}\right\rangle$ is located at the leeward edge 
that in turn represents the rapid heat removal in the streamwise direction by mean flow (Figure 8c). In the cavity, $\left\langle\theta^{\prime \prime} u^{\prime \prime}\right\rangle$ is mostly positive on the leeward side that tends to disperse hot air to the windward side, offsetting the hot air driven by the recirculation. On the other hand, it is almost zero on the windward side so the heat transfer is mainly governed by advection.

Positive vertical heat flux $\left\langle\theta^{\prime \prime} w^{\prime \prime}\right\rangle$ carries hot air upward moving away from the cavity by intermittency (Figure 8d). Its maximum, which is partly attributed to the higher temperature near the leeward rib, locates at the cavity top that signifies the rapid turbulent heat removal. Whereas, it is more uniform on the windward side that is likely a result of the mean entrainment along the windward rib.

\subsection{Coherent structures}

Quantities averaged in the spanwise direction are used in the previous sections to study the turbulent heat transfer. Additional perspective, especially the turbulent heat removal mechanism, could be accomplished by looking into the intermittency and coherent structures. These data are snapshots of the LES that are considered typical structures of flows and heat transfer.

\subsection{1. k-type flows}

Figure 9a depicts the instantaneous fluctuating flow vectors $u^{\prime \prime}$ and $w^{\prime \prime}$ and fluctuating temperature $\theta^{\prime \prime}$ on the vertical $(x-z)$ centerplane. Two instantaneous hot plumes are observed. One is over the leeward recirculation and the other is right behind the recirculation in the windward re-developing TBL. Although in different regions, they exhibit a similar coherent structure in which the hot (cold) plumes overlap with the updrafts (downdrafts). It is 
in line with the positive vertical heat flux discussed in the previous sections.

In fact, a close examination on the uprising hot plumes reveals their dissimilar characteristics and different heat removal mechanisms. First we look into the leeward recirculation $(x<5 h)$. Recalling the dividing streamline connecting the leeward edge and the reattachment point at the cavity bottom, air masses originated below (over) the dividing streamline always recirculate inside the leeward wake (go into the re-developing TBL). Therefore, the heat removal on the leeward side must be accomplished by intermittent vorticies, in pairs of updraft $\left(\theta^{\prime \prime}>0\right)$ and downdraft $\left(\theta^{\prime \prime}<0\right)$, rather than mean advection (Figure 9a). Buoyancy is not included in the current LES so the updraft and downdraft are independent from temperature difference. While the hot air masses are removed from the cavity by intermittency, cold air masses entrain into the cavity as make-up air, by intermittency, too. The intermittent flows are initiated by the unstable flow separation at the leeward edge that pairs up updraft and downdraft. These organized flows collectively carry hot air (cold make-up air) out of (into) the cavity. Likewise, LES was used in [25] to propose that Kelvin-Helmholtz instabilities render the intermittency in neutrally stratified street canyons. Their contribution to heat and mass removal of a cavity is clearly illustrated in this study.

Over the dividing streamline, no organized vortex pair is observed before the re-developing TBL. The cold air masses originated from the prevailing flow entrain into the re-developing TBL that purges the hot aged air masses out of the cavity in the streamwise direction. Concurrently, the vertical turbulent heat flux carries hot plumes out of the cavity by dispersion. Owing to the elevated edge-level turbulence, a rapid drop in temperature, in the form 
of small-scale hot air parcel, is observed right over the cavity.

The snapshots and coherent structures also help elucidate the maximum LHTC near the reattachment point at the cavity bottom. At the end of the dividing streamline, air parcels impinge on the cavity bottom and spread into two pathways moving in opposite directions: The wall jet moves toward the leeward rib then carries hot air out of the cavity following the recirculating flows. Simultaneously, the re-developing TBL drives hot air masses by mean flow. Their collective heat removal eventually leads to the local maximum of LHTC near the reattachment point.

\subsection{2. d-type flows}

Figure $9 \mathrm{~b}$ shows the intermittent flow vectors and hot plumes on the vertical $(x-z)$ centerplane in $d$-type flows. In this narrow cavity of $h / b=1 / 4$, the re-developing TBL vanishes. Instead of touching down the cavity bottom, the dividing streamline bridges the leeward and windward ribs covering the cavity so the heat removal is solely driven by intermittency. In the lower half of the cavity, hot air is carried by the wall jet in the recirculation toward the leeward rib, then moving upward for heat removal. Vortex pairs, which are initiated by flow instability, are also observed after the leeward rib, suggesting the intermittent hot air removal and cold air entrainment.

\section{Conclusions}

LES is sought to examine the heat removal mechanisms of the $k$ - and $d$ type flows over a cavity flanked by a pair of ribs. The air and heat exchanges in the two flow regimes are contrasted by means of dividing streamline. 
The $k$-type flow is characterized by the recirculation and re-developing TBL, respectively, on the leeward and windward sides. The dividing streamline, originated from the top of the leeward rib, descends and finally impinges on the cavity bottom at $x=5 h$, resulting in the maximum LHTC. Air masses below the dividing streamline remain inside the leeward recirculation. Hot air, in the form of updraft, is removed from the recirculation by intermittent vortex pairs. Likewise, cold air follows the downdraft migrating into the recirculation. The flow instability at the leeward rib generates the vortex pairs. Air masses over the dividing streamline, which are the cool air originated from the prevailing flow, are carried by the air entrainment into the re-developing TBL of the cavity. As a result, the hot air is removed by the purging cold air toward the windward side in the streamwise direction.

The heat in $d$-type flow is removed by a different mechanism. The dividing streamline does not touch the cavity bottom but covers the leeward and windward ribs. The recirculation thus occupies the entire cavity that is isolated from the prevailing flow. The heat removal from the cavity is therefore totally governed by intermittent vortex pairs generated at the leeward edge. The LHTC is monotonic increasing from the leeward to the windward sides.

\section{Acknowledgments}

Two anonymous reviewers provided constructive suggestions. This project was supported by the General Research Fund of the Hong Kong Research Grant Council HKU 715209E. The computation is supported in part by a HKUGC Special Equipment Grant (SEG HKU09). The technical support from Lilian Y.L. Chan, Frankie F.T. Cheung, and W.K. Kwan with Com- 
puter Centre, The University of Hong Kong is appreciated.

\section{References}

[1] R. L. Haugen, A. M. Dhanak, Heat transfer in turbulent boundary-layer separation over a surface cavity, J. Heat Transfer 89 (1967) 335-340.

[2] J. Jimenez, Turbulent flows over rough walls, Ann. Rev. Fluid Mech. 36 (2004) 143-196.

[3] T. R. Oke, Street design and urban canopy layer climate, Energy Bldg. 11 (1988) 103-113.

[4] E. L. Jarrett, T. L. Sweeney, Mass transfer in rectangular cavities, AIChE Journal 13 (1967) 797-800.

[5] D. E. Metzger, R. S. Bunker, M. K. Chyu, Cavity heat transfer on a transverse grooved wall in a narrow flow channel, J. Heat Transfer 111 (1989) 73-79.

[6] E. M. Sparrow, W. Q. Tao, Enhanced heat transfer in a flat rectangular duct with streamwise periodic disturbances at one principal wall, J. Heat Transfer 105 (1983) 851-861.

[7] E. A. Sewall, D. Tafti, A. B. Graham, K. A. Thole, Experimental validation of large eddy simulations of flow and heat transfer in a stationary ribbed duct, Int. J. Heat Fluid Flow 27 (2006) 243-258.

[8] T. J. Young, K. Vafai, Experimental and numerical investigation of forced convective characteristics of arrays of channel mounted obstacles, J. Heat Transfer 121 (1999) 34-42. 
[9] P. Promvonge, C. Thianpong, Thermal performance assessment of turbulent channel flows over different shaped ribs, Int. Commun. Heat Mass Transf. 35 (2008) 1327-1334.

[10] J. F. Barlow, I. N. Harman, S. E. Belcher, Scalar fluxes from urban street canyons. Part I: Laboratory simulation, Boundary-Layer Meteorol. 113 (2004) 369-385.

[11] K. Narita, Experimental study of the transfer velocity for urban surfaces with a water evaporation method, Boundary-Layer Meteorol. 122 (2007) 293-320.

[12] A. Hagishima, J. Tanimoto, K.-I. Narita, Intercomparisons of experimental convective heat transfer coefficients and mass transfer coefficients of urban surfaces, Boundary-Layer Meteorol. 117 (2005) 551-576.

[13] A. Saidi, B. Sunden, Numerical simulation of turbulent convective heat transfer in square ribbed ducts, Numer. Heat Transf., A Appl. 38 (2000) $67-88$.

[14] H.-M. Kim, K.-Y. Kim, Shape optimization of three-dimensional channel roughened angled ribs with RANS analysis of turbulent heat transfer, Int. J. Heat Mass Transfer 49 (2006) 4013-4022.

[15] E. Elsaadawy, H. Mortazavi, M. S. Hamed, Turbulence modeling of forced convection heat transfer in two-dimensional ribbed channels, J. Electron. Packaging 130 (2008) 1-17. 
[16] D. N. Ryu, D. H. Choi, V. C. Patel, Analysis of turbulent flow in channels roughened by two-dimensional ribs and three-dimensional blocks. Part I: Resistance, Int. J. Heat Fluid Flow 28 (2007) 1098-1111.

[17] D. N. Ryu, D. H. Choi, V. C. Patel, Analysis of turbulent flow in channels roughened by two-dimensional ribs and three-dimensional blocks. Part II: Heat transfer, Int. J. Heat Fluid Flow 28 (2007) 1111-1124.

[18] A. K. Viswanathan, D. K. Tafti, Detached eddy simulation of turbulent flow and heat transfer in a ribbed duct, ASME J. Fluids Eng. 127 (2005) 888-896.

[19] A. Ashrafian, H. I. Andersson, M. Manhart, DNS of turbulent flow in a rod-roughened channel, Int. J. Heat Fluid Flow 25 (2004) 373-383.

[20] A. Ashrafian, H. I. Andersson, The structure of turbulence in a rodroughened channel, Int. J. Heat Fluid Flow 27 (2006) 65-79.

[21] S. Leonardi, P. Orlandi, L. Djenidi, R. A. Antonia, Structure of turbulent channel flow with square bars on one wall, Int. J. Heat Fluid Flow 25 (2004) 384-392.

[22] Y. Nagano, H. Hattori, T. Houra, DNS of velocity and thermal fields in turbulent channel flow with transverse-rib roughness, Int. J. Heat Fluid Flow 25 (2004) 393-403.

[23] C.-H. Liu, M. C. Barth, Large-eddy simulation of flow and scalar transport in a modeled street canyon, J. Applied Meteoro. 41 (2002) 660-673. 
[24] Z. Q. Cui, X. M. Cai, C. J. Baker, Large-eddy simulation of turbulent flow in a street canyon, Q. J. Roy. Meteor. Soc. 130 (2004) 1373-1394.

[25] M. O. Letzel, M. Krane, S. Raasch, High resolution urban large-eddy simulation studies from street canyon to neighbourhood scale, Atmos. Environ. 36 (2001) 8770-8784.

[26] T. N. H. Chung, C.-H. Liu, Pollutant transfer coefficient in street canyons of different aspect ratios, in: 13th International Conference on Harmonisation within Atmospheric Dispersion Modelling for Regulatory Purposes, Paris, France, 2010.

[27] OpenFOAM, Openfoam: The open source CFD toolbox, http://www.openfoam.com/ (2010).

[28] J. Smagorinsky, General circulation experiments with the primative equations I: The basic experiment, Month. Weath. Rev. 91 (1963) 99165.

[29] T.-M. Liou, J.-J. Hwang, S.-H. Chen, Simulation and measurement of enhanced turbulent heat transfer in a channel with periodic ribs on one principal wall, Int. J. Heat Mass Transfer 36 (1993) 507-517.

[30] D. B. Spalding, A new analytical expression for the drag of a flat plate valid for both the turbulent and laminar regimes, J. Heat and Mass Transfer 5 (1962) 1133-1138.

[31] B. E. Launder, D. B. Spalding, The numerical computation of turbulent flows, Comput. Methods Appl. Mech. Eng. 3 (1974) 269-289. 
[32] R. Goldstein, H. Cho, A review of mass transfer measurements using naphthalene sublimation, Exp. Therm. Fluid Sci. 10 (1995) 416-434.

[33] F. P. Incropera, Fundamentals of Heat and Mass Transfer, sixth Edition, John Wiley, Hoboken, NJ, 2007.

[34] D. Aliaga, J. Lamb, D. Klein, Convection heat transfer distribution over plates with square ribs from infrared thermography measurements, Int. J. Heat Mass Transfer. 37 (1994) 363-374.

[35] H. Yamamoto, N. Seki, S. Fukusako, Forced convection heat transfer on heated bottom surface of a cavity, J. Heat Transfer 101 (1979) 475-479.

[36] S. Acharya, S. Dutta, T. A. Myrum, R. S. Baker, Periodically developed flow and heat transfer in a ribbed duct, Int. J. Heat Mass Transfer 36 (1993) 2069-2082.

[37] R. Kiml, A. Magda, S. Mochizuki, A. Murata, Rib-induced secondary flow effects on local circumferential heat transfer distribution inside a circular rib-roughned tube, Int. J. Heat Mass Transfer 47 (2004) 14031412.

[38] E. Leriche, S. Gavrilakis, Direct numerical simulation of the flow in a lid-driven cubical cavity, Phys. Fluids A12 (2000) 1363-1376.

[39] S. E. Belcher, Mixing and transport in urban areas, Phil. Trans. R. Soc. A 363 (2005) 2947-2968. 


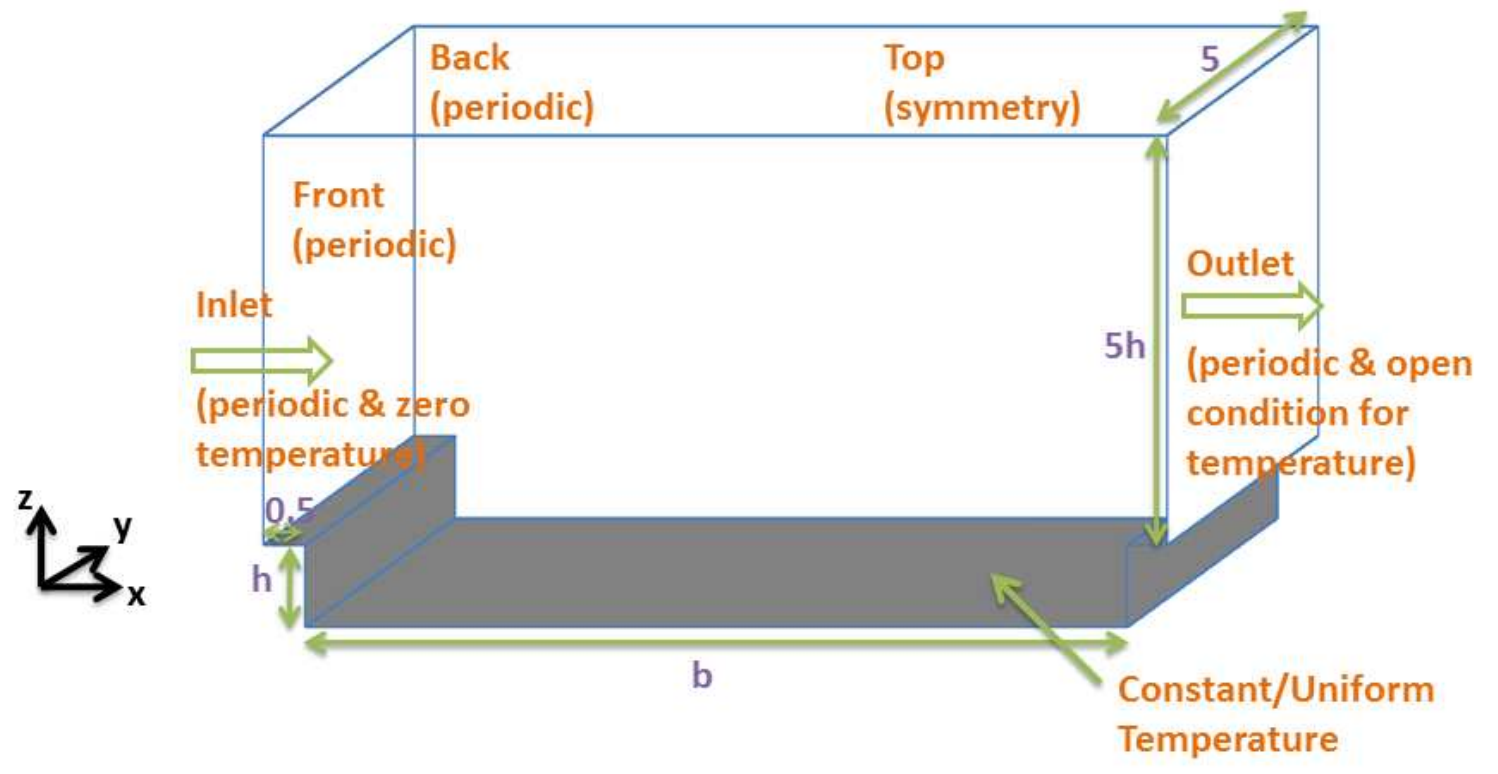

Figure 1: Computational domain of the LES. 
Nomenclature

$b \quad$ rib separation

$c, m, n$ empirical constants for heat transfer over ribs

$\mathrm{C}_{k}, \mathrm{C}_{\epsilon} \quad$ empirical modeling constants, $\mathrm{C}_{k}=0.07$ and $\mathrm{C}_{\epsilon}=1.05$

$\mathrm{E} \quad$ empirical constant in logarithmic wall model, $=0.9$

$h$ rib height

$k \quad$ turbulence kinetic energy (TKE)

$\mathrm{N} \quad$ number of elements

$\mathrm{Nu} \quad$ Nusselt number

Pr Prandtl number, $=0.72$

$p \quad$ kinematic pressure

$q_{\text {cond }} \quad$ heat flux by conduction

$q_{\text {conv }} \quad$ heat flux by forced convection

Re Reynolds number

Sc $\quad$ Schmidt number

$x_{i} \quad$ spatial tensor

$x, y, z$ Cartesian coordinates in streamwise, spanwise, and wall-normal directions

$\mathrm{U} \quad$ prevailing wind speed

$u_{i} \quad$ velocity vector

$u, v, w$ velocity components in streamwise, spanwise, and wall-normal directions

$u_{\tau} \quad$ friction velocity, $=\sqrt{\tau / \rho}$ where $\rho$ is fluid density

Greek symbols

$\alpha \quad$ molecular thermal diffusivity, $=\nu / \operatorname{Pr}=\mathcal{K} /\left(\rho \mathrm{C}_{\mathrm{p}}\right)$ where $\mathcal{K}$ and $\mathrm{C}_{\mathrm{p}}$ are thermal conductivity and specific heat at constant pressure of fluid, respectively

$\Delta \quad$ filter width, $=\left(\Delta_{x} \Delta_{y} \Delta_{z}\right)^{1 / 3}$

$\Delta P \quad$ background kinematic pressure gradient

$\Delta \Theta \quad$ temperature difference, $=\Theta_{h}-\Theta_{c}$

$\delta_{i j} \quad$ Kronecker delta

$\gamma_{i} \quad$ subgrid-scale heat flux, $=\overline{\theta u_{i}}-\bar{\theta} \bar{u}_{i}$

$\kappa \quad$ von Kármán constant, $=0.42$

$\nu \quad$ kinematic viscosity

$\omega \quad$ local heat transfer coefficient, $=q_{\text {conv }} /(\mathrm{U} \Delta \Theta)$

$-\tau_{i j} \quad$ Reynolds stress, $=-\left(\bar{u}_{i} u_{j}-\bar{u}_{i} \bar{u}_{j}\right)$

$\Theta_{c} \quad$ temperature in the background

$\Theta_{h} \quad$ temperature on cavity bottom and rib surfaces

$\theta \quad$ temperature

Superscripts

$+\quad$ properties expressed in wall unit

$\phi^{\prime \prime} \quad$ deviation from the ensemble average, $\phi^{\prime \prime}=\bar{\phi}-\langle\bar{\phi}\rangle$

$\hat{\phi} \quad$ unit vector in the wall-normal direction

$\bar{\phi} \quad$ resolved-scale properties in large-eddy simulation

Subscripts

\| properties parallel to a wall

$i, j \quad$ indices used in tensor notation, $=1,2,3$

$\perp \quad$ properties normal to a wall

SGS subgrid-scale (SGS) properties

Symbols

$\langle\phi\rangle \quad$ properties averaged in the homogeneous spanwise direction 

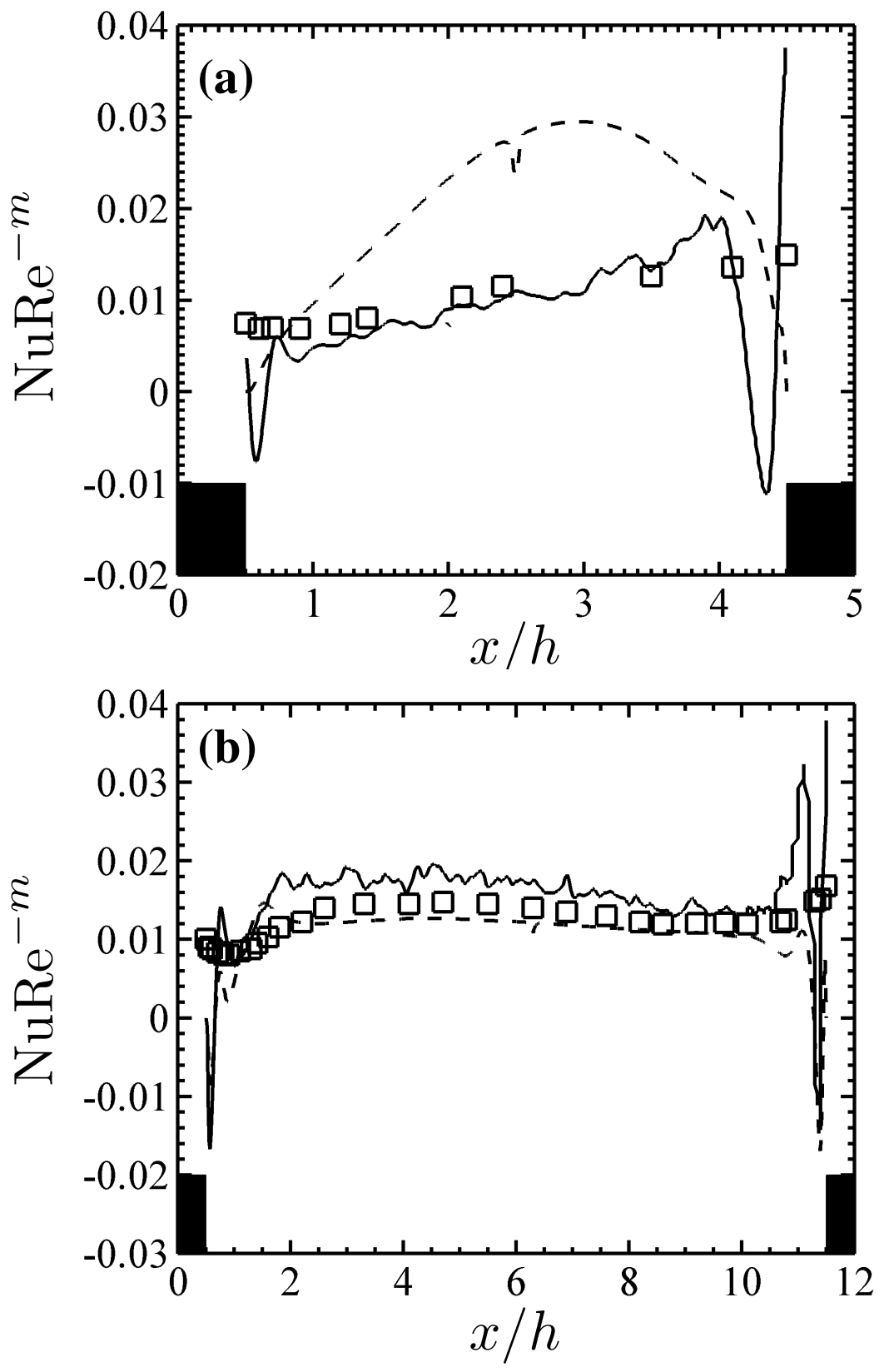

Figure 2: Dimensionless LHTC at the cavity bottom in-between a pair of ribs of $h / b=$ : (a). $1 / 4$ ( $d$-type flow) and (b). $1 / 11$ ( $k$-type flow). $\square$ : Experiment [34]; - - - $k-\epsilon$ turbulence model; and - : LES. 

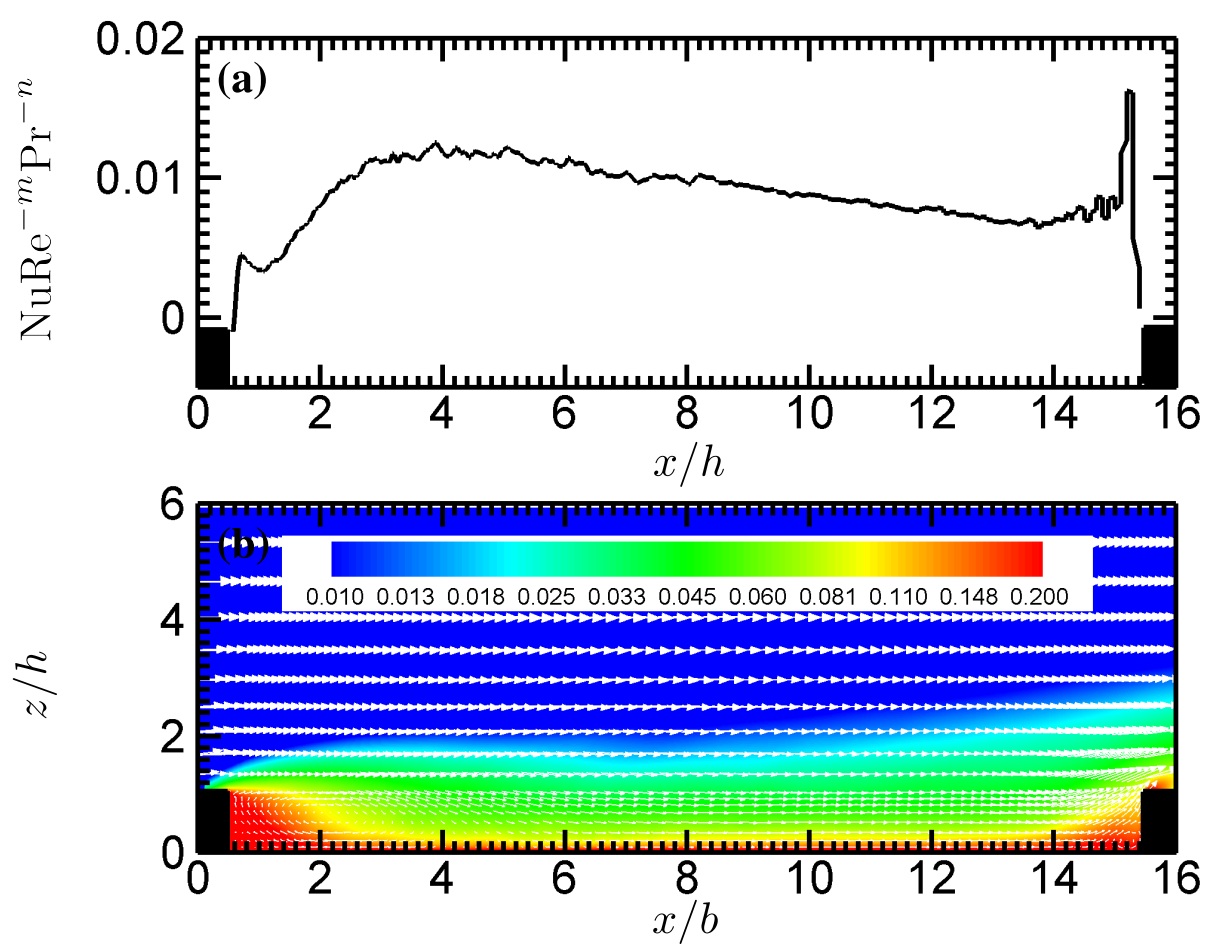

Figure 3: (a). Dimensionless LHTC at the cavity bottom and (b). velocity vectors in-between a pair of ribs of $h / b=1 / 15$ ( $k$-type flow). Also shown in (b) are the contours of dimensionless temperature $\langle\bar{\theta}\rangle / \Delta \Theta$. 

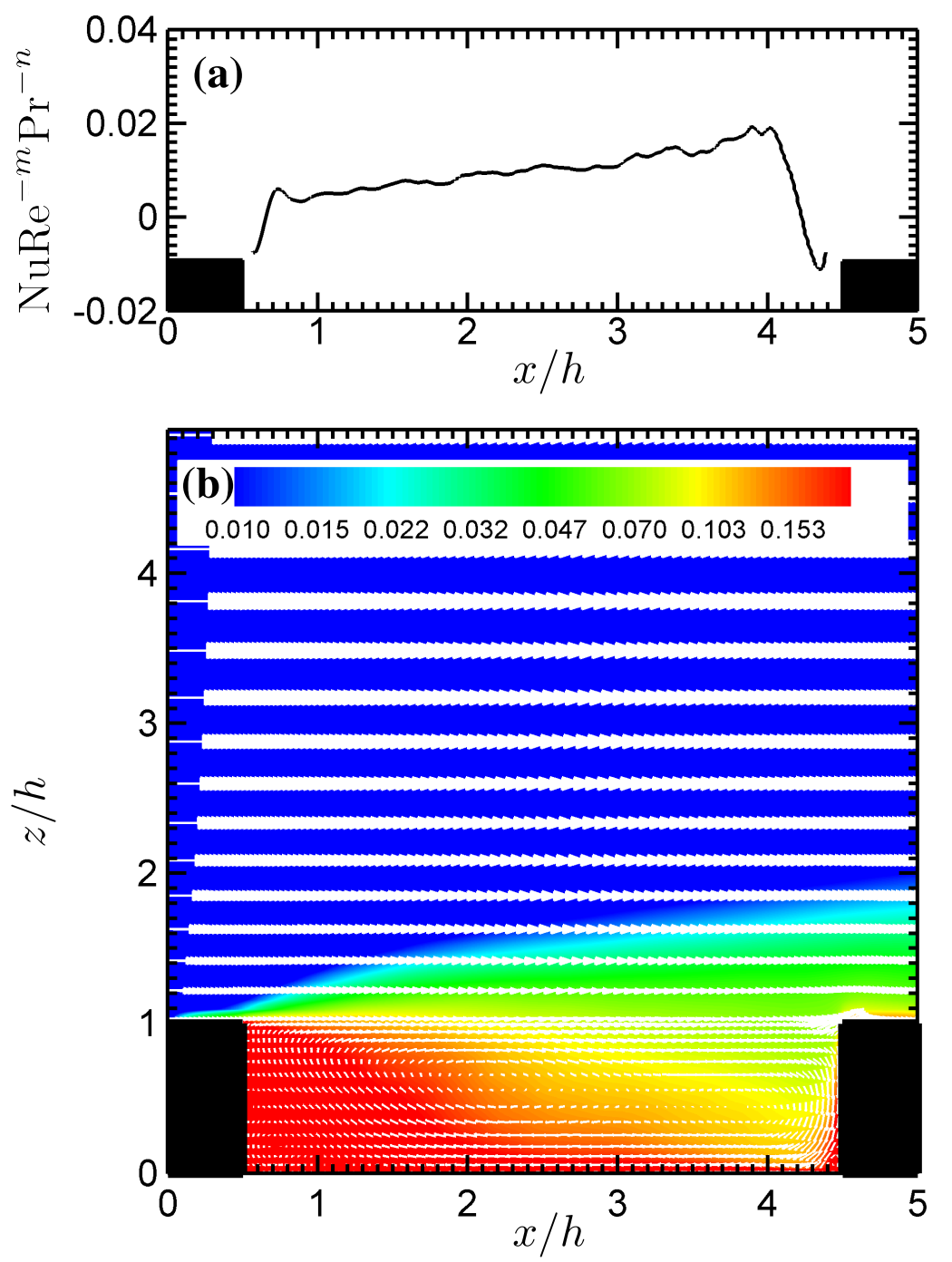

Figure 4: (a). Dimensionless LHTC at the cavity bottom and (b). velocity vectors in-between a pair of ribs of $h / b=1 / 4$ ( $d$-type flow). Also shown in (b) are the contours of dimensionless temperature $\langle\bar{\theta}\rangle / \Delta \Theta$. 

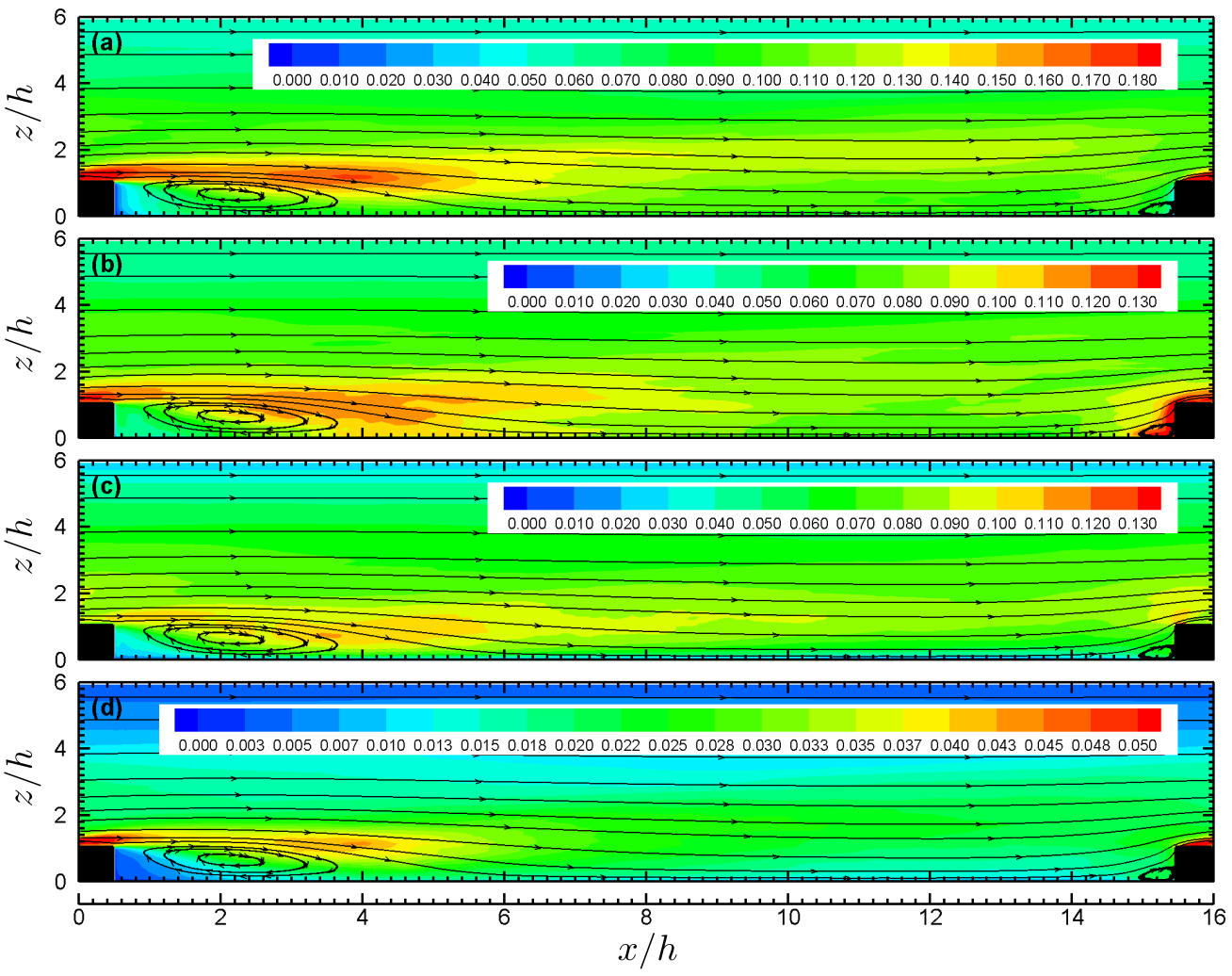

Figure 5: Contours of the turbulence structures in the cavity of $h / b=1 / 15$. (a). $\left\langle u^{\prime \prime} u^{\prime \prime}\right\rangle^{1 / 2} / \mathrm{U}_{0} ;$ (b). $\left\langle v^{\prime \prime} v^{\prime \prime}\right\rangle^{1 / 2} / \mathrm{U}_{0} ;(\mathrm{c}) .\left\langle w^{\prime \prime} w^{\prime \prime}\right\rangle^{1 / 2} / \mathrm{U}_{0}$; and (d). $\operatorname{TKE}\left(=\left\langle u^{\prime \prime} u^{\prime \prime}+v^{\prime \prime} v^{\prime \prime}+w^{\prime \prime} w^{\prime \prime}\right\rangle / 2 / \mathrm{U}_{0}\right)$. Also shown are the streamlines. 

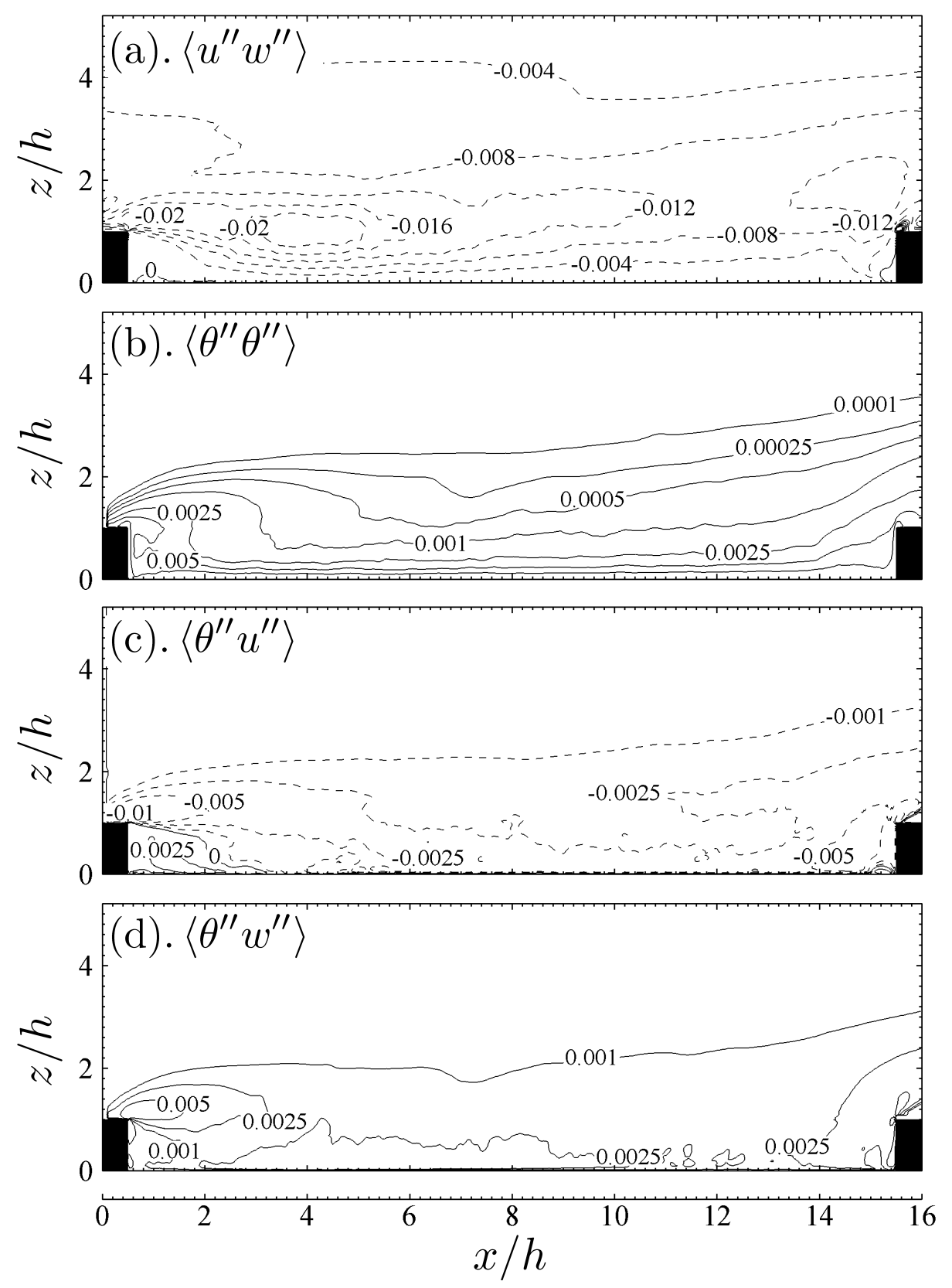

Figure 6: Contours of (a). vertical momentum flux $\left\langle u^{\prime \prime} w^{\prime \prime}\right\rangle$; (b). temperature covariance $\left\langle\theta^{\prime \prime} \theta^{\prime \prime}\right\rangle$; (c). streamwise $\left\langle\theta^{\prime \prime} u^{\prime \prime}\right\rangle$; and (d). vertical $\left\langle\theta^{\prime \prime} w^{\prime \prime}\right\rangle$ directions. 

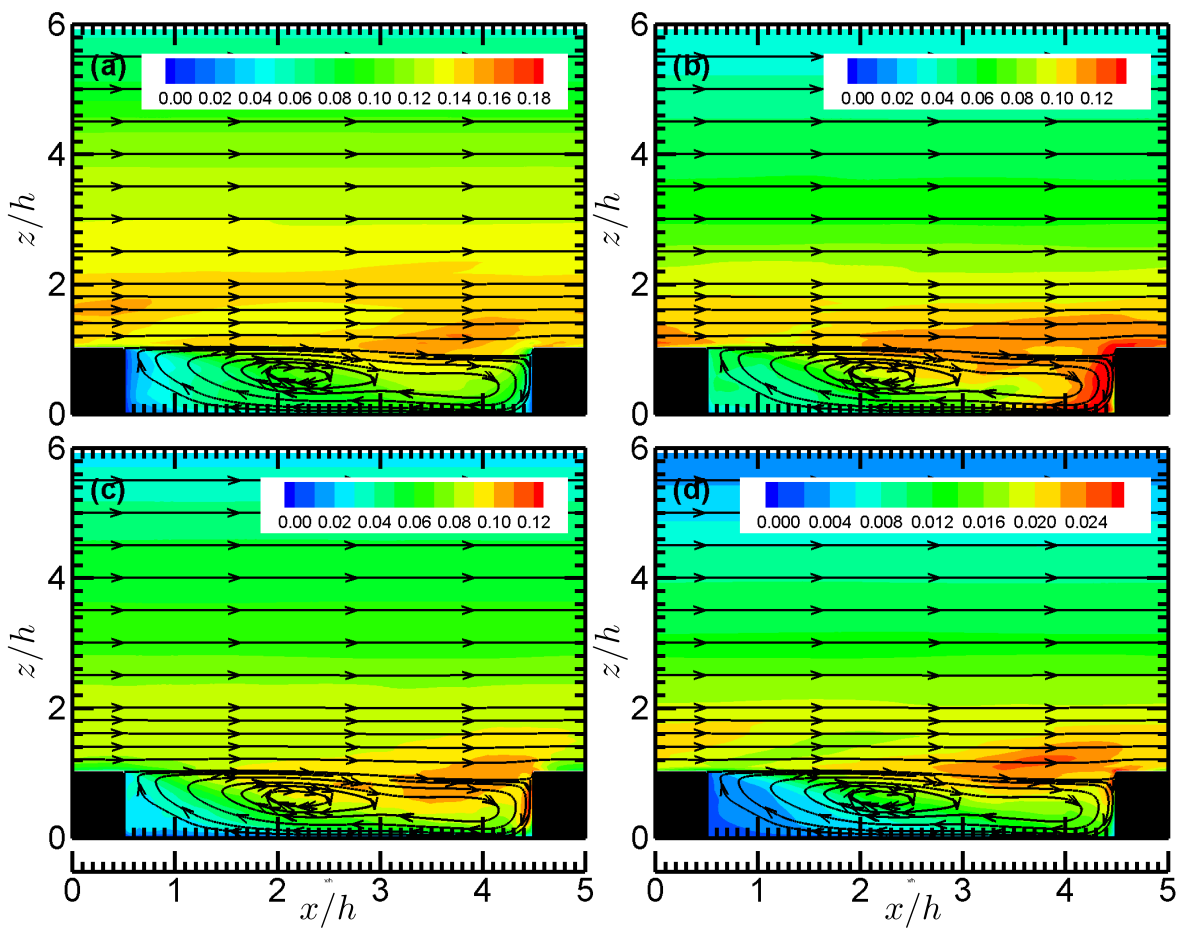

Figure 7: Contours of the turbulence structures in the cavity of $h / b=1 / 4$.

(a). $\left\langle u^{\prime \prime} u^{\prime \prime}\right\rangle^{1 / 2} / \mathrm{U}_{0} ;$ (b). $\left\langle v^{\prime \prime} v^{\prime \prime}\right\rangle^{1 / 2} / \mathrm{U}_{0} ;(\mathrm{c}) .\left\langle w^{\prime \prime} w^{\prime \prime}\right\rangle^{1 / 2} / \mathrm{U}_{0}$; and (d). $\operatorname{TKE}\left(=\left\langle u^{\prime \prime} u^{\prime \prime}+v^{\prime \prime} v^{\prime \prime}+w^{\prime \prime} w^{\prime \prime}\right\rangle / 2 / \mathrm{U}_{0}\right)$. Also shown are the streamlines. 

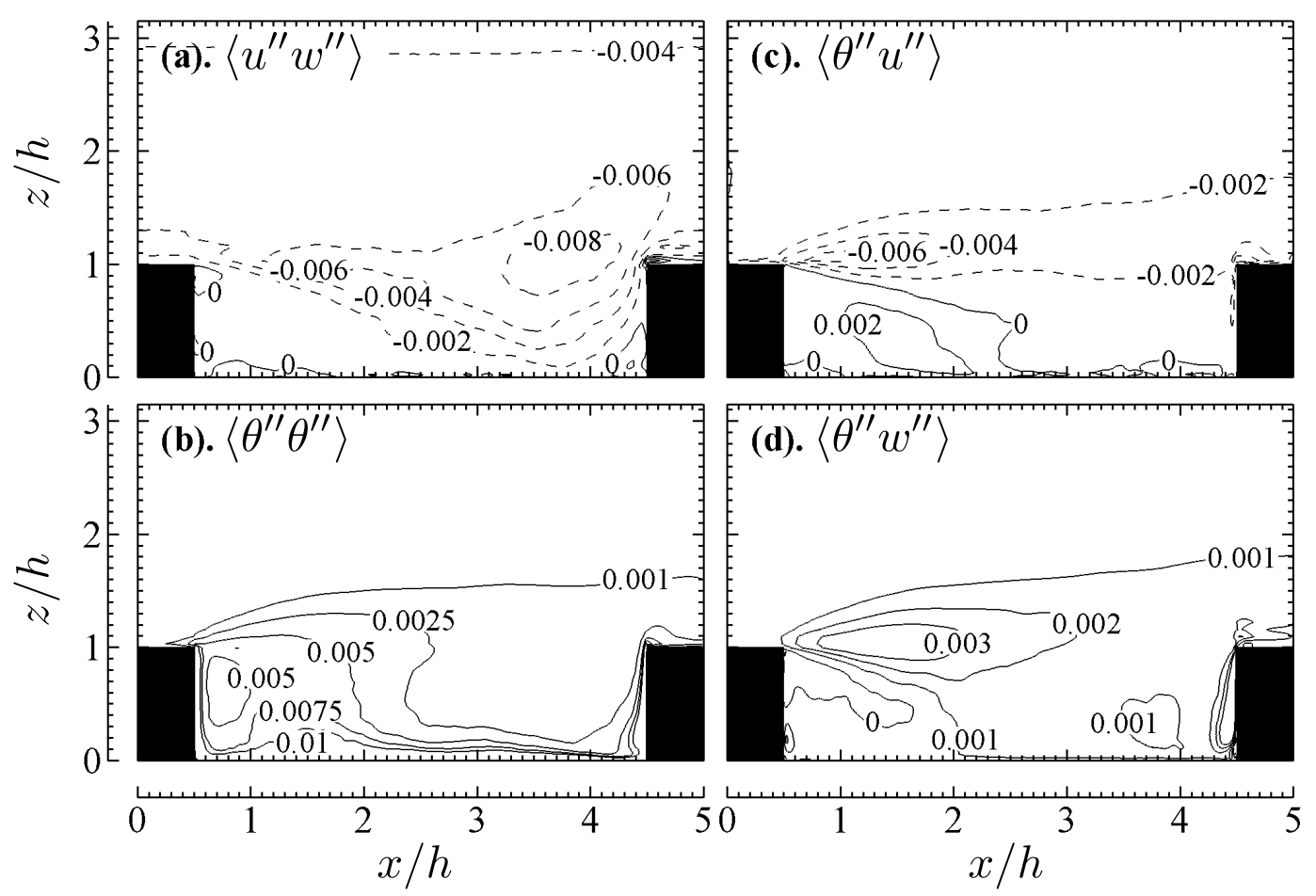

Figure 8: Contours of (a). Momentum flux $\left\langle u^{\prime \prime} w^{\prime \prime}\right\rangle$, (b). temperature covariance $\left\langle\theta^{\prime \prime} \theta^{\prime \prime}\right\rangle$, (c). streamwise heat flux $\left\langle\theta^{\prime \prime} u^{\prime \prime}\right\rangle$ and (d). vertical heat flux $\left\langle\theta^{\prime \prime} w^{\prime \prime}\right\rangle$. 

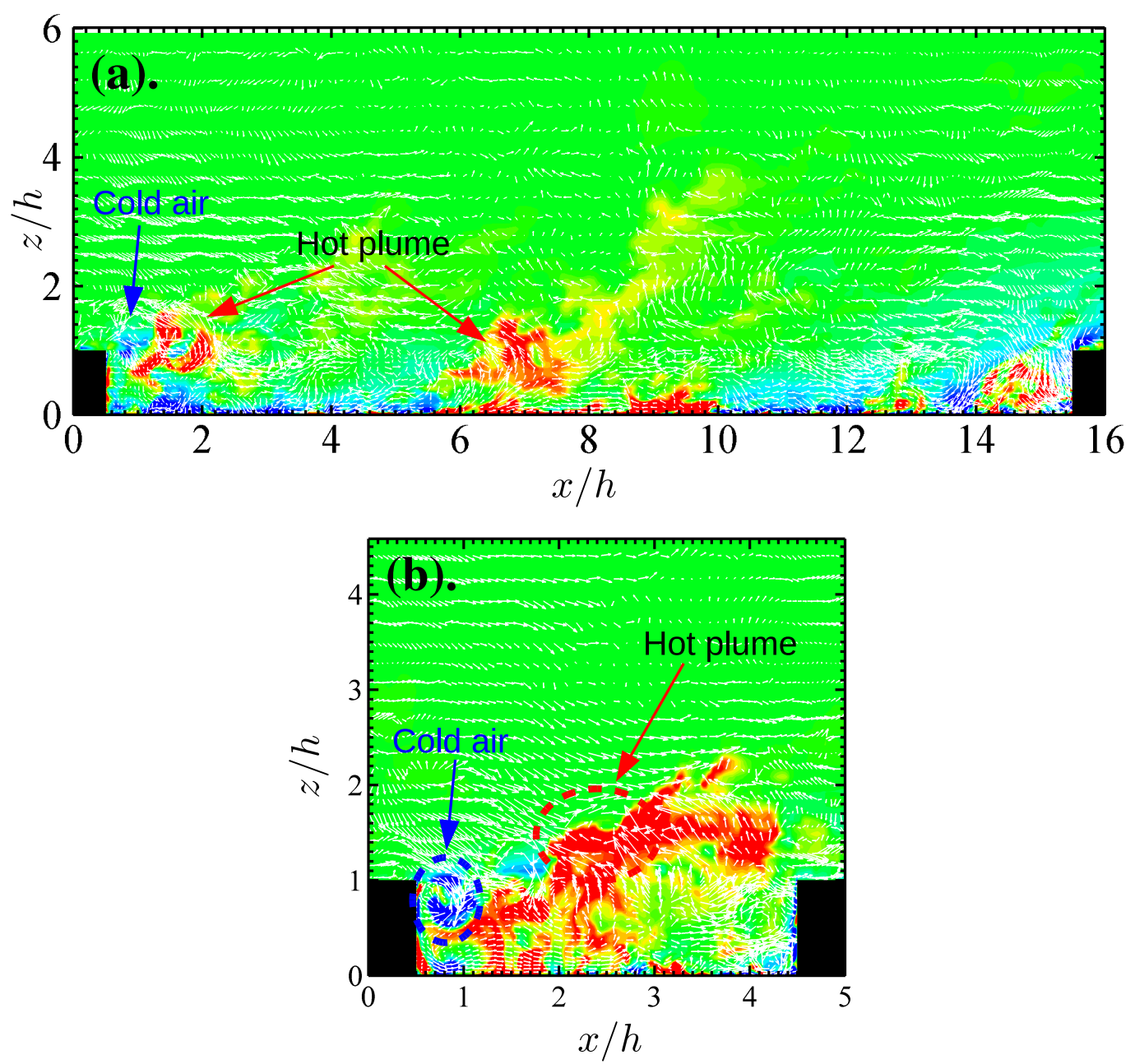

Figure 9: Snapshot of temperature and flow vectors of a cavity in-between a pair of ribs of $h / b=$ : (a). 1/15 ( $k$-type flows) and (b). $1 / 4$ ( $d$-type flows). 